\title{
Determinants of Postural Orientation in Quadrupedal Stance
}

\author{
Joyce Fung and Jane M. Macpherson \\ R. S. Dow Neurological Sciences Institute, Portland, Oregon 97209
}

The purpose of this study was to investigate the determinants of postural orientation by examining stance kinematics and kinetics at various interpaw distances. Four adult cats were trained to stand, unrestrained, on a force platform. Three-dimensional ground reaction forces and kinematics, as well as EMG activities, were recorded during stance at five different anteroposterior (AP) distances and two widths. Stance distance was varied by changing the distance between the four force plates mounted on the platform.

All cats used a strategy of maintaining a constant horizontal orientation of the trunk and levering the limbs at the girdles while malntalning constant Intralimb geometry to accommodate the changes in stance distance. The direction of the ground reaction forces covaried with the limb axes. As a result, the joint torques were conserved in the forelimb, and varied within small ranges in the hind. Increased tonic activity in the extensors of the back, the hip, and the ankle was observed at shorter distances while increased knee extensor activity was observed at longer distances. A cost function, defined as the sum of squared 3-D joint torques, was minimal for the hindlimb at a stance distance which corresponded to the preferred distance naturally assumed by each cat on the floor. Thus, in the maintenance of stance posture, trunk orientation and iritralimb geometry is constrained, the goal of which is to minimize muscular effort or energy expenditure.

[Key words: stance, posture, cat, biomechanics, kinetics, kinematics, EMG]

It is not a simple task, from a physiological point of view, for a terrestrial animal to maintain posture and orientation through changing terrains. The maintenance of stance requires not only that the body be supported against gravity, but also that the body's center of mass remain within the limits of support in the horizontal plane. Quadrupeds have an intrinsically stable musculoskeletal framework (Gray, 1944). However, there are control parametcrs and ncural mechanisms which may be defincd within the context of mechanical constraints, in order to counteract internal and external forces, and to orient the animal for food and movement. Movements of any body segment must be properly

Received May 13, 1994; revised July 13, 1994; accepted July 25, 1994.

The very able technical assistance of Dr. Charles Russell, Ms. Robyn Crandell, and Mi. John Knight is gratefully acknowledged. The helpful and stimulating discussions with Drs. Ron Jacobs and Arthur Kuo are kindly appreciated. This work was supported by a grant to J.M.M. from the NIH (NS29025) and a fellowship to J.F. from the Rick Hansen Man in Motion Foundation of Canada.

Correspondence should be addressed to Jane Macpherson, Ph.D., RSDNSI, 1120 NW 20th Avenue, Portland, OR 97209.

Copyright (C) 1995 Society for Neuroscience $0270-6474 / 95 / 151121-11 \$ 05.00 / 0$ counterbalanced to preserve equilibrium. Stance maintenance is a dynamic task in which sensorimotor systems must interact with the external environment, to maintain stability and proper alignment of the limbs, trunk, and head-neck system.

Stabilization of the trunk in space may be the major controlled variable in postural orientation and equilibrium, since the bulk of the body's mass resides in the trunk. The trunk is oriented and controlled by the action of the limbs exerting force against the support surface. A previous study (Macpherson, 1994) has shown that interpaw distance has a significant effect on the forces exerted by the cat both during quiet stance and during perturbation of the support surface, which may have an impact on trunk stability. It was postulated that at long stance distances, the force constraint strategy (Macpherson, 1988) was mechanically useful in preventing bending movements of the spine, whereas at short stance distances, trunk stability was provided by tonic activation of the epaxial trunk muscles.

Roberts (1973) proposed, primarily through behavioral observation in animals, that vestibular and neck reflexes may interact to produce appropriate tonic muscle activities in the limbs to maintain trunk stability during stance on uneven or inclined surfaces. Gurfinkcl et al. (1981) also suggested that in humans, an important goal for postural regulation is to align the trunk with respect to earth's vertical. This is based on the constant phase relations observed between trunk displacement and EMG changes when subjects were tilted rhythmically at the support surface at different frequencies. However, by subjecting cats to various degrees of platform tilt during quiet stance, Lacquaniti et al. $(1984,1990)$ concluded that orienting the limbs with respect to earth's vertical is the essential goal of postural control.

This study addresses the determinants of postural orientation by examining the kinematics, kinetics, and muscle activity of cats during quiet stance at various interpaw distances. In theory, there are two global strategies which can be adopted by the freely standing cat as stance distance changes: either (1) constrain the orientation of the trunk and incline the limbs, or (2) maintain a constant vertical alignment of the limbs and change the curvature of the spine. It will be shown that the first strategy, namely, orienting the trunk and not the limbs in space, is the major postural constraint for stance maintenance.

\section{Materials and Methods}

General procedures. Four adult cats (GR, MI, PR, and LU) with body weights ranging from 3.7 to $4.4 \mathrm{~kg}$ were trained, using food reinforcement, to stand quietly on a force platform. The platform consisted of a metal surface $(46 \times 25 \mathrm{~cm})$ on which four triaxial force plates were fixated by magnets and double-sided tape. The distance between the paws was varied by adjusting the position of the force plates on the platform (Fig. 1). The "preferred" stance distance was determined from the natural paw-separation distance assumed by each cat on the floor. A mean of 10 measurements was taken as the cat stood intermittently still while roaming freely over the tiled floor of the laboratory. As crude 
A. Sagittal View

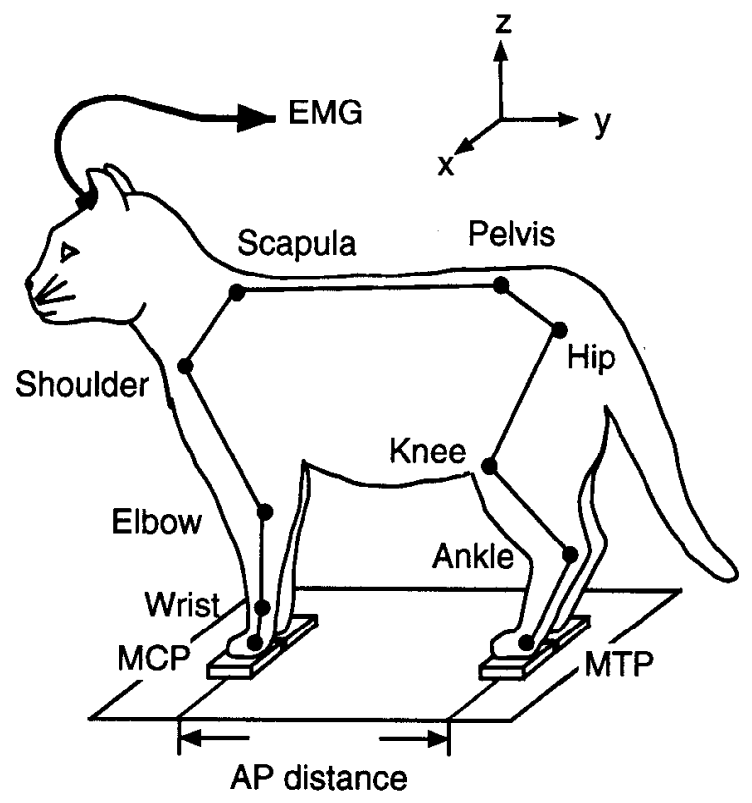

B. Frontal View

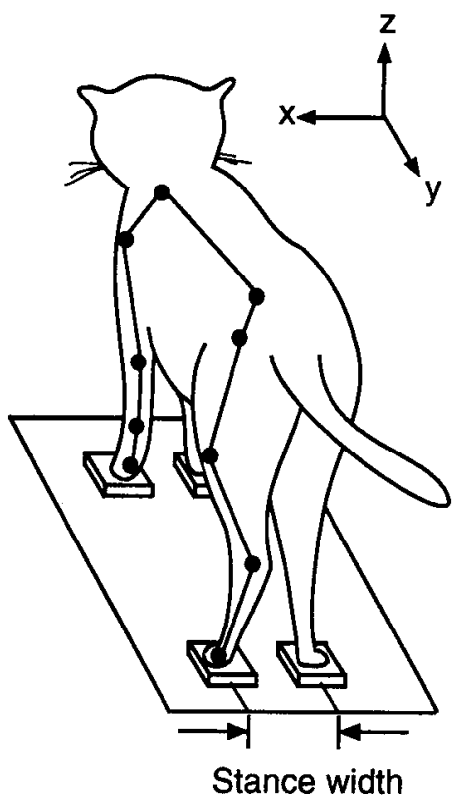

Figure 1. Schematic of the cat standing on a force platform in two different views: sagittal $(A)$ and frontal $(B)$. AP distance and stance width were varied by changing the distance between the force plates. The kinematics and ground reaction forces in three orthogonal directions are specified by the $x-y-z$ axes of the coordinate system shown.
This frequency was selected on the basis of a residual analysis of the preliminary data.

Data analysis. Force, EMG, and joint position data were averaged over the 100 msec epochs ( $n=40-80$ per set). Joint radius, skin depth, and IRED base thickness were subtracted from the x-positions to approximate joint centers of rotation. The actual fore-hindpaw separation distance was obtained by calculating the distance between the metacarpophalangeal (MCP) and metatarsophalangeal (MTP) joint markers. This was termed anteroposterior (AP) distance and was used subsequently as the independent variable in all regression analyses.

Forces were analyzed in the three cardinal planes (sagittal, frontal, horizontal) as well as in 3-D. The vertical (Fv), longitudinal (Fy), and lateral $(\mathrm{Fx})$ force components under each paw were summed in pairs to obtain the planar vectors. Center of pressure (CoP) in the AP and lateral directions was calculated by summing, respectively, Fvs of the forepaws and Fvs of the right paws, with each normalized as a percentage of the summed Fvs of all four paws. Under static conditions and averaged over time, the $\mathrm{CoP}$ is equivalent to the position of the center of mass projected onto the horizontal plane.

Joint angles and torques were computed in the cardinal planes as well as in 3-D. Joint torques were computed using inverse dynamics equations, the parameters of which, including the center of mass and moments of inertia of body segments, were estimated from regression equations (Hoy and Zernicke, 1985) using known measures of body weight and segment length. The algorithms for the computation and batch processing of joint torques were developed by Dr. R. Jacobs in the laboratory using MATLAB (Mathworks).

Force, kinematic, and kinetic variables were graphed against AP distance as scatter plots and smoothed by a function using locally weighted least sums of squares (LOWESS). A tension of 0.8 was used in all the LOWESS smoothing procedures. The LOWESS lines were helpful in determining the choice of curve fitting. When the LOWESS line appeared linear, a linear equation was fitted to the data. When the line appeared nonlinear, a model of parameter optimization was used to estimate the constants in the nonlinear equation.

Analyses of variance (ANOVAs) were used to determine if there were any main effects of AP distance and width on dependent variables such as CoP, GRF magnitude, joint angle, and joint torque. In such instances, three blocks of the AP distance (shortest vs preferred vs longest) or two levels of width ( 8 vs $12 \mathrm{~cm}$ ) were used as the independent variable. The order of set was entered as a covariate to control for any adaptation effects over time. Tukey test of pairwise comparisons was done post hoc when a significant main effect existed. A $p$ level of less than 0.01 was accepted as significant. All statistical and smoothing procedures were done using SYSTAT (Systat). 


\section{A. Body Geometry}

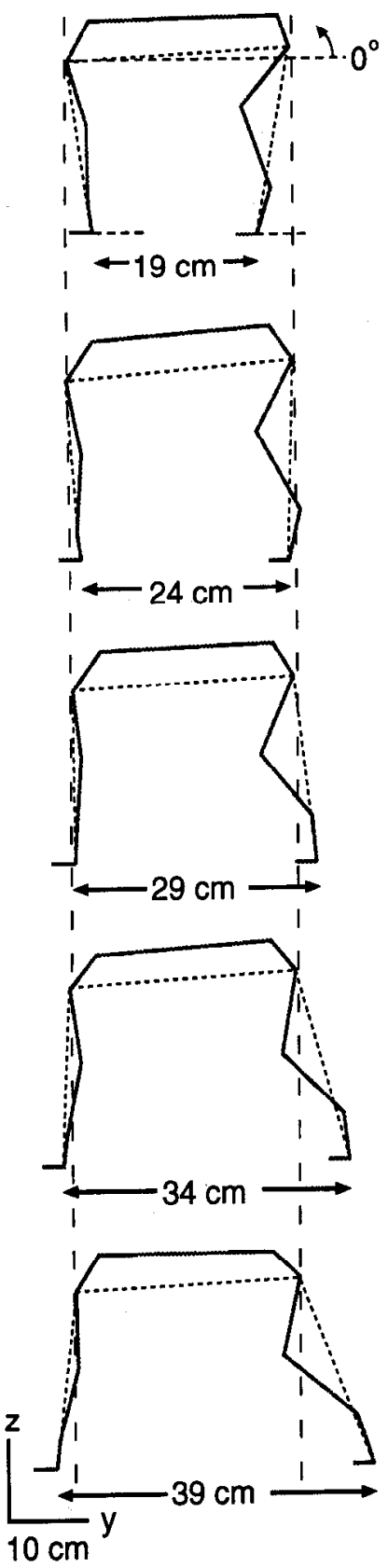

\section{B. Hindlimb Axis}

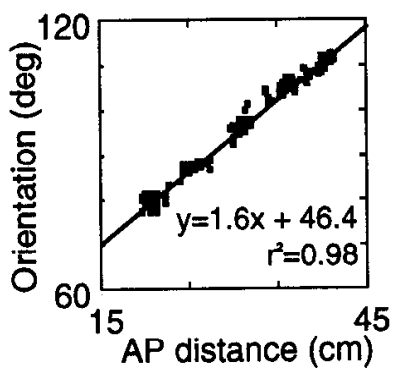

\section{Forelimb Axis}

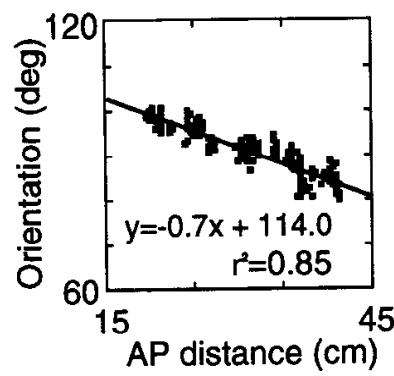

D. Trunk Axis

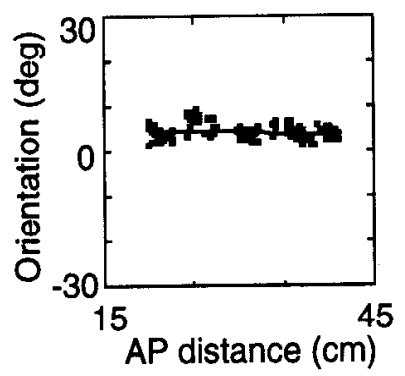

E. Girdle Height

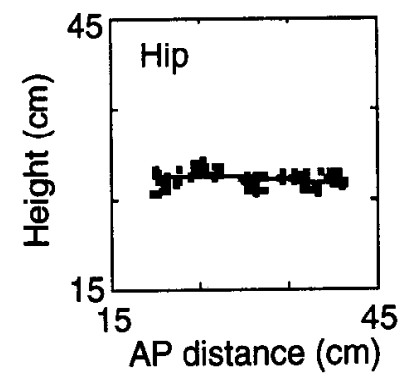

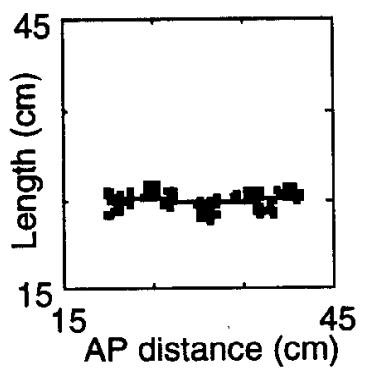
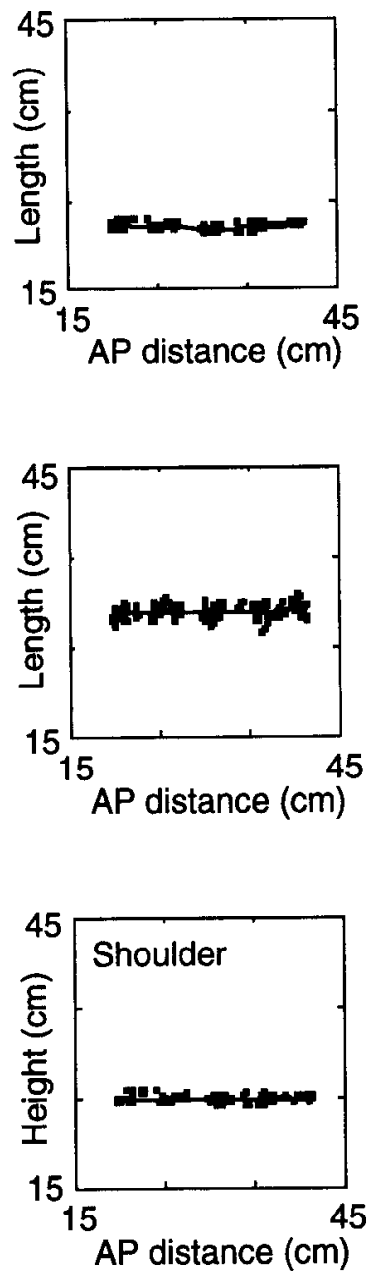

Figure 2. A, Body geometry (from cat GR) in sagittal view shown as stick figures representing the mean joint positions at different fore-hindpaw separation distances. The hindlimb axis and forelimb axis are shown as dotted lines joining the paws and the girdles, whereas the trunk axis is shown as a dotted line joining the girdles at the hip and shoulder. Vertical dashed lines through the figurines represent the earth's vertical reference. Note the constant trunk alignment and levering of the limb axes with increase in stance distance. Orientation was measured from the horizontal $\left(0^{\circ}\right)$, as illustrated in the top stick figure. $B-D$, Sagittal orientation and length of the hindlimb axis $(B)$, forelimb axis $(C)$, and trunk axis $(D)$, plotted against the actual AP distance measured between the MCP and MTP. Note the linear relation between the orientation of limb axes and AP distance (described by the regression equation and $r^{2}$ ). A constant orientation and length of the trunk is shown by the LOWESS lines through the scatter plots. $E$, Height of the girdles measured as distances from hip and shoulder to the support surface plotted against AP distance.

\section{Results}

Due to the larger range of distances tested, the fore-hindpaw separation distance along the sagittal axis had a more profound effect on the biomechanics of quiet stance than the change in width. along the transverse axis. The results due to fore-hind distance variation will be presented first, followed by the effects of width variation.

\section{Effects due to fore-hind distance changes}

General body posture. All four cats showed a strategy of changing limb orientation and constraining trunk geometry to accommodate changes in fore-hind stance distance. A representative set of trunk-limb configurations at different fore-hind distances

from Cat GR is illustrated in Figure $2 A$. Each stick was plotted from the $\mathrm{y}-\mathrm{z}$ positions averaged across 80 trials (100 msec each in duration) of quiet stance. It can be seen that the hindlimb axis (defined as the straight line between the MTP and hip joint) changed its direction markedly from inclining backward at the shortest stance distance to inclining forward at the longest stance distance. An opposite effect on orientation was observed in the forelimb axis (defined as the straight line between the MCP and shoulder joint), although the range of directional changes was much smaller than that observed in the hindlimb. As further illustrated in the scatter plots in Figure 2, $B$ and $C$, these directional changes were linearly related to the actual fore-hind AP distance (between MCP and MTP). The linear relations were 
strong in the hindlimb, with $r^{2}$ ranging from 0.94 to 0.98 in all four cats, indicating that AP distance could account for almost the entire variance in limb axis orientation. With the exception of cat LU $\left(r^{2}=0.62\right)$, the linear relations were also moderately strong in the forelimb, having $r^{2}$ that ranged from 0.85 to 0.87 in the other three cats. It is noteworthy that in all the cats examined, the hindlimb axis was most vertically aligned at an AP distance intermediate between the shortest and preferred stance, while the forelimb axis was most vertical at the preferred stance distance.

In contrast, the trunk axis (defined as the straight line between the shoulder and hip joint) remained nearly horizontally aligned (Fig. 24), with its orientation and length invariant across all $\Lambda \mathrm{P}$ distances (Fig. 2D). In fact, the length of the trunk axis was related to the length of the preferred stance distance, in that cats which had longer trunk axes also preferred longer fore-hind stance distances. The girdle heights, measured by the vertical distances from hip and shoulder to the support surface, also remained constant at all distances (Fig. $2 E$ ). This observation illustrates that the limbs were used as levers pivoting at their articulations with the trunk, while trunk geometry was being conserved to accommodate changes in stance distance. No marked changes were observed in the length of the limb axes (Fig. 2B,C). The apparent discrepancy between the invariance in girdle height on the one hand and the invariance in limb length on the other may be explained in terms of trigonometry. A $10^{\circ}$ inclination of the limb from vertical would result in no more than a $1.5 \%$ increase in length if girdle height remains constant, that is, a $4 \mathrm{~mm}$ increase in the length of the limb axis for a vertical distance of $250 \mathrm{~mm}$. Such a small change would not be evident given the range of variability in the data.

Paw-ground contact forces. The ground reaction force (GRF) vector is equal in magnitude and opposite in direction to the force exerted by the paw. The sagittal and frontal plane GRFs at different fore-hind stance distances are plotted in relation to limb gcometry for the left hindlimb (Fig. $3 A, B$ ) and for the left forelimb (Fig. 4A,B) from cat GR. As stance distance increased, the sagittal plane GRF in the hindlimb also changed direction markedly, from a nearly vertical orientation at the shortest distance to a forward inclination at the longest distance (Fig, 3A). A reversed directional change was observed in the sagittal forelimb GRF (Fig. 4A). The directional changes were linearly related to the AP distance (Figs. $3 C, 4 C$ ), with $r^{2}$ ranging from 0.93 to 0.97 for the hind and from 0.91 to 0.97 for the fore in all four cats. It is noteworthy that the GRF was most vertically oriented at a distance intermediate between the shortest and the preferred for both the hindlimb (Fig. 3A,C) and forelimb (Fig. $4 A, C$ ). This coincided with the vertical limb axis orientation in the hind but not in the fore (see previous section).

The directional change in GRF was also linearly related to the directional change in limb axis (Figs. $3 E, 4 E$ ), with $r^{2}$ in all the cats ranging from 0.94 to 0.96 in the hind and from 0.80 to 0.95 in the fore. In the hindlimb, the change in GRF direction was smaller than the change in limb axis orientation (Fig. 3E), whereas in the forelimb, GRF direction was closely matched with limb axis orientation (Fig. $4 E$ ). This is also indicated by the slopes of the linear equations, which ranged from 0.4 to 0.6 in the hind and from 0.9 to 1 in the fore. However, with the effects of AP distance partialcd out, the residual correlation between limb axis and GRF orientations was poor for the hindlimb ( $r_{y \cdot x}$ ranging from 0.41 to 0.57 ) and moderate for the forelimb ( $r_{y \cdot x}$ ranging from 0.68 to 0.83 ).

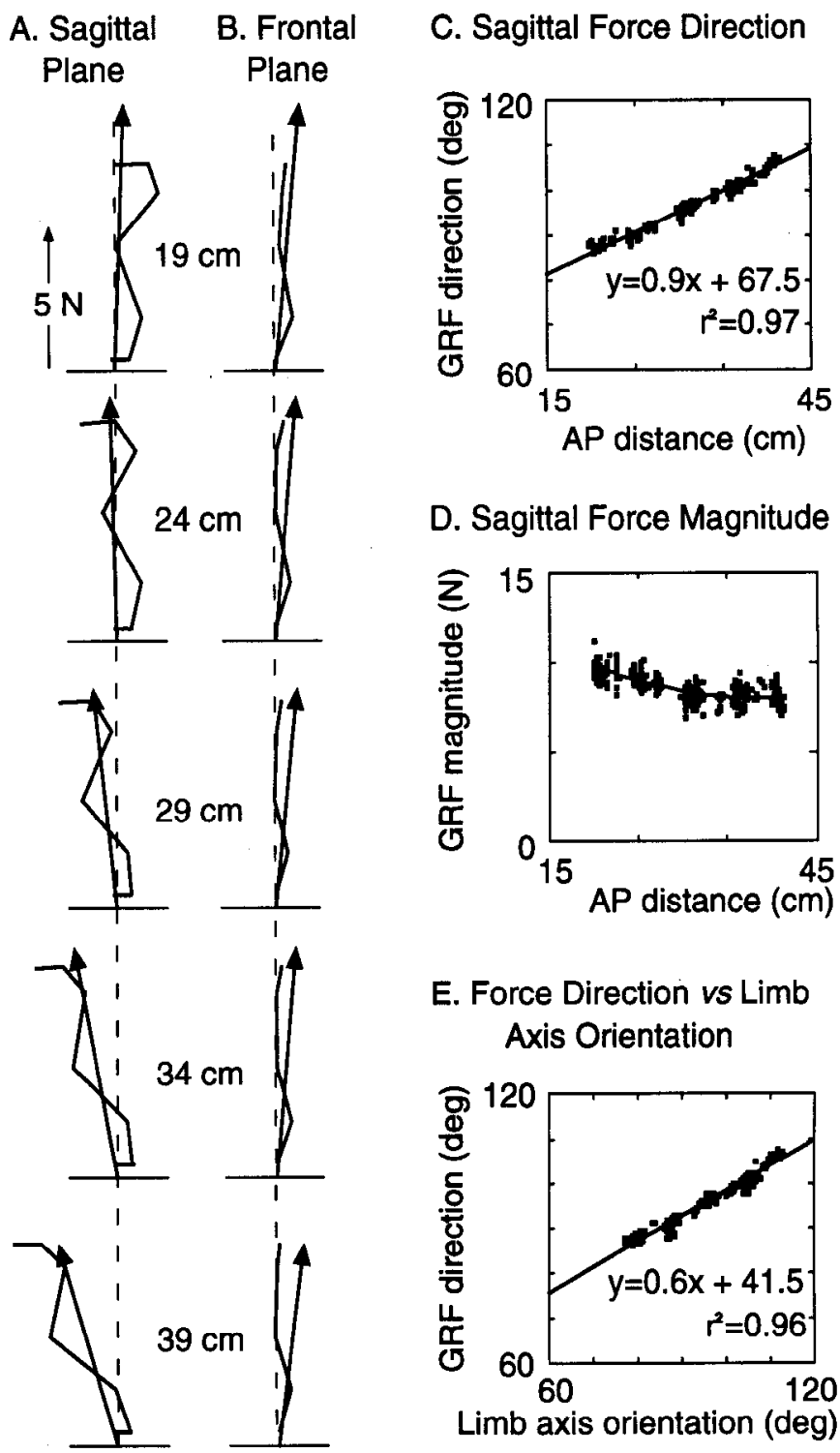

Figure 3. Hindlimb ground reaction force. The mean position of force vector (from cat GR) and its relation to the left hindlimb at different fore-hind stance distances plotted in the sagittal $(A)$ and frontal $(B)$ planes. Dashed lines represent the vertical reference through the origin of the force vector. Note the change in GRF vector direction in the sagittal plane in relation to the vertical as well as the hip joint $(A) . C$, Direction and $D$, magnitude of the sagittal force vector plotted against AP distance. Note the linear relation of vector direction to AP distance $(C)$ represented by the regression equation. $E$, GRF direction plotted against orientation of the hindlimb axis.

As far as the magnitude of the sagittal GRF is concerned, there was a trend of decrease in the hind (Fig. 3D) and increase in the fore (Fig. 4D) when AP distance increased. This might be due to a shift of weight from the hind to the fore as the hindlimb levered backwards. Indeed, the AP CoP showed a significant forward shift from the shortest stance distance to the preferred and from the preferred to the longest stance distance. This finding was common to all four cats.

In the frontal plane, no significant correlation was found between the direction of GRF or limb axis with AP distance changes. The limb configuration was closely aligned with the vertical at all distances for both the hind (Fig. $3 B$ ) and the fore 


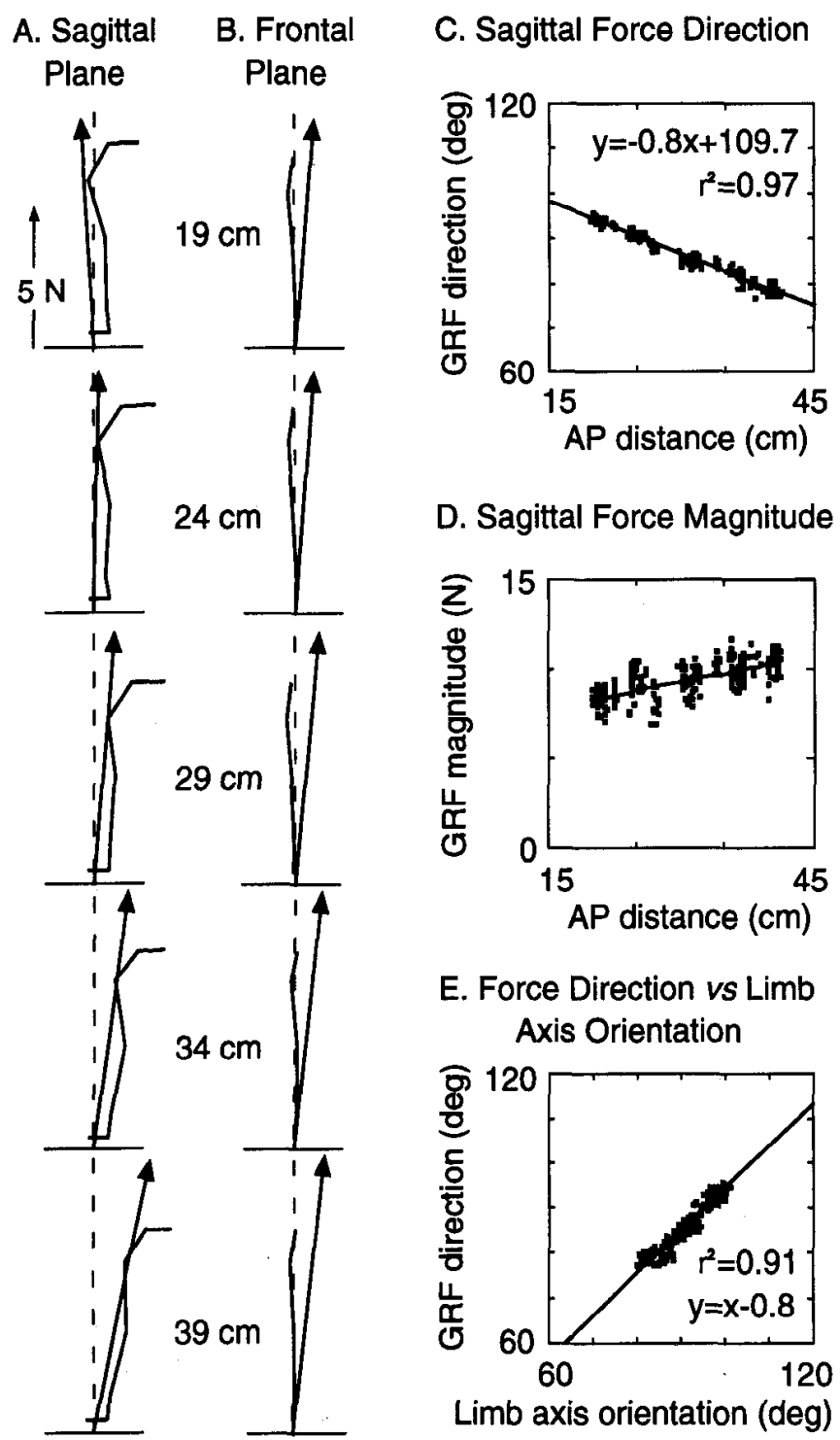

Figure 4. Forelimb ground reaction force. The mean position of force vector (from cat GR) and its relation to the left forelimb at different fore-hind stance distances plotted in the sagittal $(A)$ and frontal $(B)$ planes. Dashed lines represent the vertical reference through the origin of the force vector. Note the change in vector direction in the sagittal plane in relation to the vertical as well as the shoulder joint $(A) . C$, Direction and $D$, magnitude of the sagittal force vector plotted against AP distance. Note the linear relation of vector direction to AP distance (C) represented by the regression equation. $E$, GRF direction plotted against orientation of the forelimb axis. A linear relation with a slope of 1 was observed, indicating a close alignment of the force vector with the limb axis.

(Fig. 4B), while the GRF was directed inward with a slight increase in inclination only at the longest stance distance.

The horizontal plane forces constituted an interesting pattern (Fig. 5A) which changed markedly with fore-hindpaw separation distance as previously reported (Macpherson, 1994). At the preferred stance distance ( $29 \mathrm{~cm}$ in Fig. $5 A$ ), the forces were diagonally directed, such that the hindpaws exerted forces backward and outward. As stance distance lengthened (34 and 39 $\mathrm{cm}$ ), the forces became more sagittally directed. As stance distance shortened, the forces became more laterally directed and reversed along the sagittal axis direction at the shortest distance

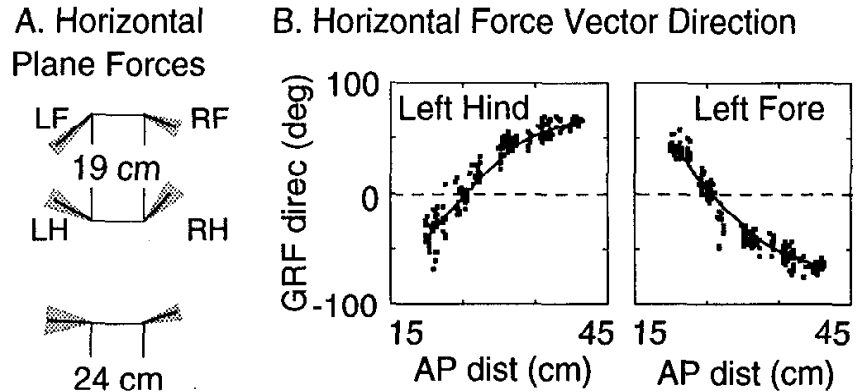

C. Horizontal Force Vector Magnitude
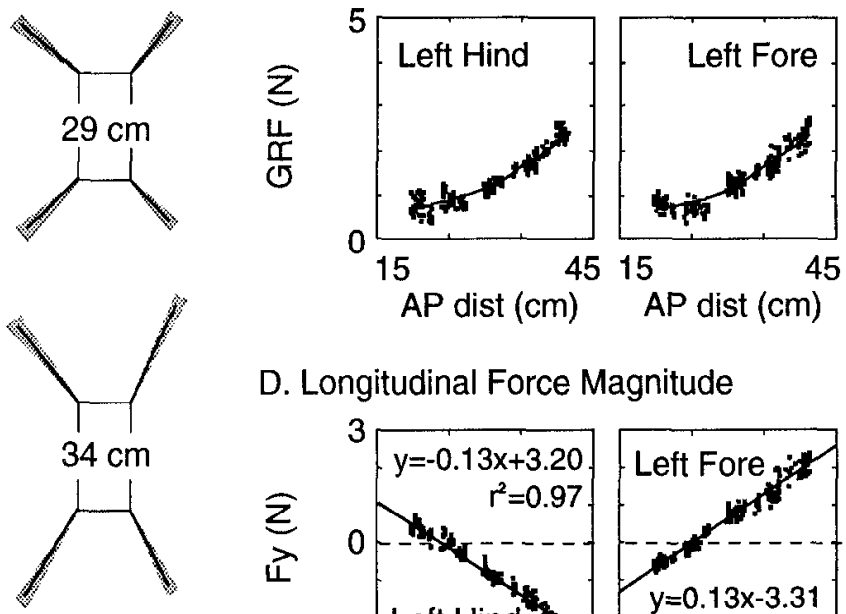

D. Longitudinal Force Magnitude
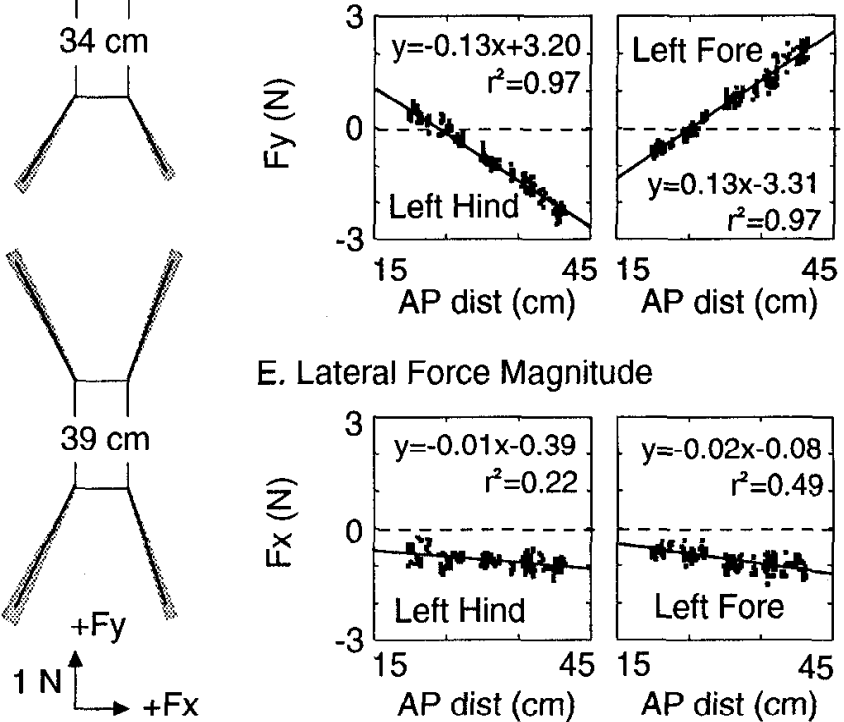

E. Lateral Force Magnitude

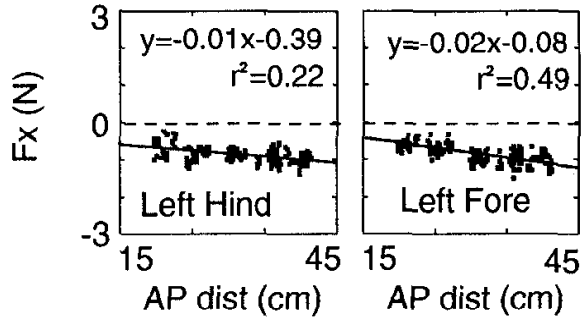

Figure 5. Horizontal plane forces. A, Planar representation of shear forces exerted by each limb at different fore-hind stance distances. Solid lines emanating from corners of rectangles represent mean vector direction and relative amplitude; surrounding shaded areas indicate \pm 1 $\mathrm{SD}$. The coordinate system for the force components is shown below the bottom figure. $B$, Direction of the horizontal plane GRF (opposite to the force exerted by the paw) plotted against AP distance. $C$, Magnitude of the horizontal plane GRF plotted against AP distance. Note curvilinear trends depicted by the LOWESS lines in all the scatter plots $(B, C) . D$, Magnitude of the longitudinal $(F y)$ and lateral $(F x)$ compo nents of the horizontal forces. Note the larger changes in Fy as compared to Fx with AP distance changes.

$(19 \mathrm{~cm})$. The directional change in horizontal GRF showed a curvilinear relation with AP distance (Fig. $5 B$ ), such that a linear trend was observed at shorter stance distances and the slope became more gentle at longer distances. The horizontal GRF magnitude also showed a curvilinear trend of increase with AP distance (Fig. $5 \mathrm{C}$ ), but the slope was steeper at longer rather than shorter distances. A closer examination of the individual 
A. Hindlimb Segment Angles: sagittal
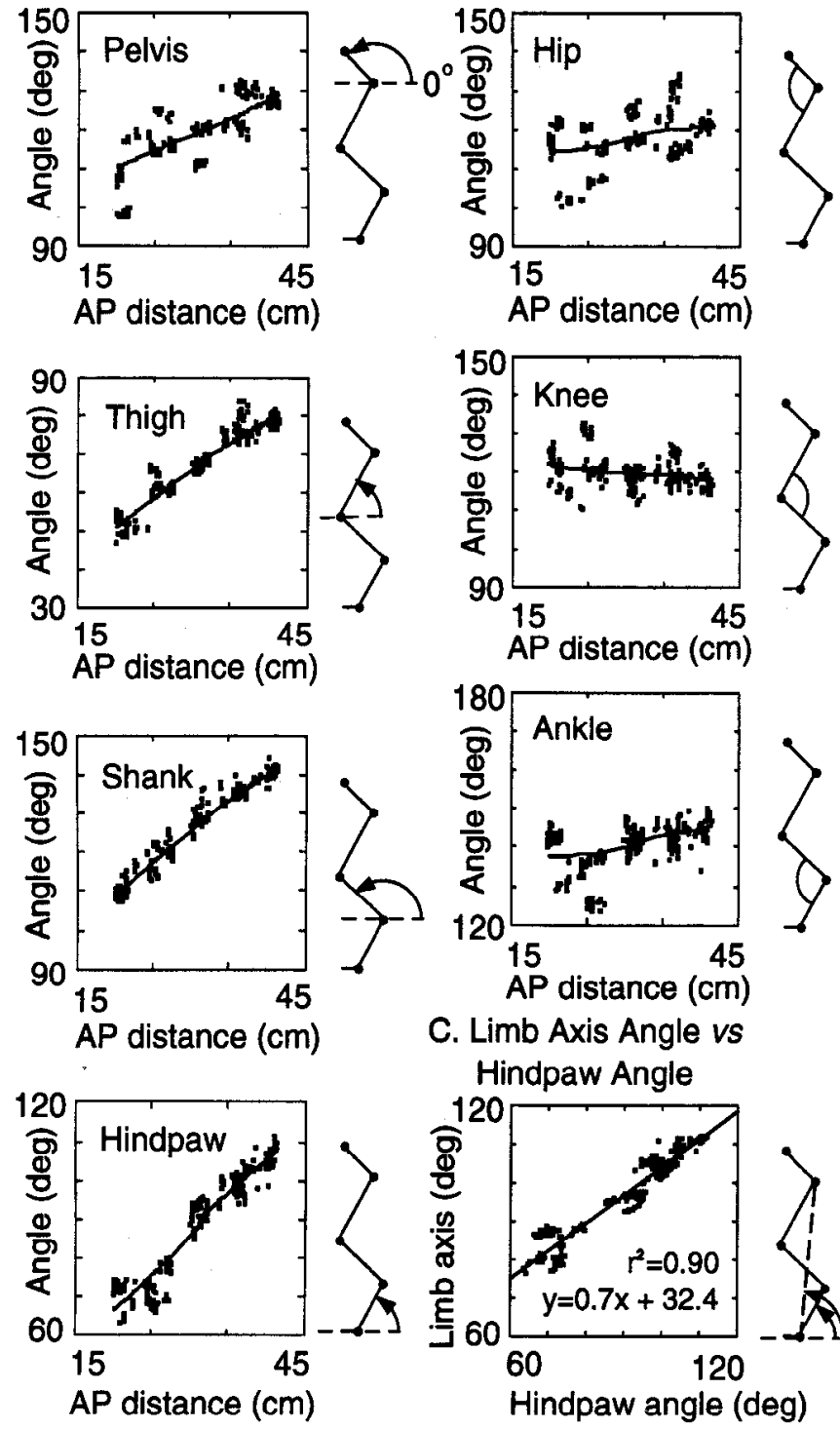

Figure 6. Stance kinematics of the hindlimb. A, Sagittal segment angles plotted against AP distance, with superimposed LOWESS lines indicating linear relations. Figures on the side indicate the angular coordinate system $\left(0^{\circ}\right.$ horizontal). $B$, Sagittal joint angles plotted against AP distance with superimposed LOWESS lines. Figures on the side indicate the measured angle, with the direction of increase indicating extension. $C$. Direction of hindlimb axis plotted against hindpaw angle with respect to horizontal.

force components showed a remarkable linear relation of the longitudinal component (Fy) with AP distance changes (Fig. $5 D$ ), while the lateral component ( $F x)$ remained relatively unchanged.

Limb kinematics. The effects of AP distance on kinematics are shown in Figure 6 for the hindlimb and in Figure 7 for the forelimb. Cat GR was again chosen as an example for the purpose of illustration, to relate to the previous figures on body posture and GRF, but the general findings were common to all four cats. As shown in Figure 6A, the sagittal plane hindlimb segment angles increased markedly with the increase in AP distance. The relations were all linear, as shown by the LOWESS
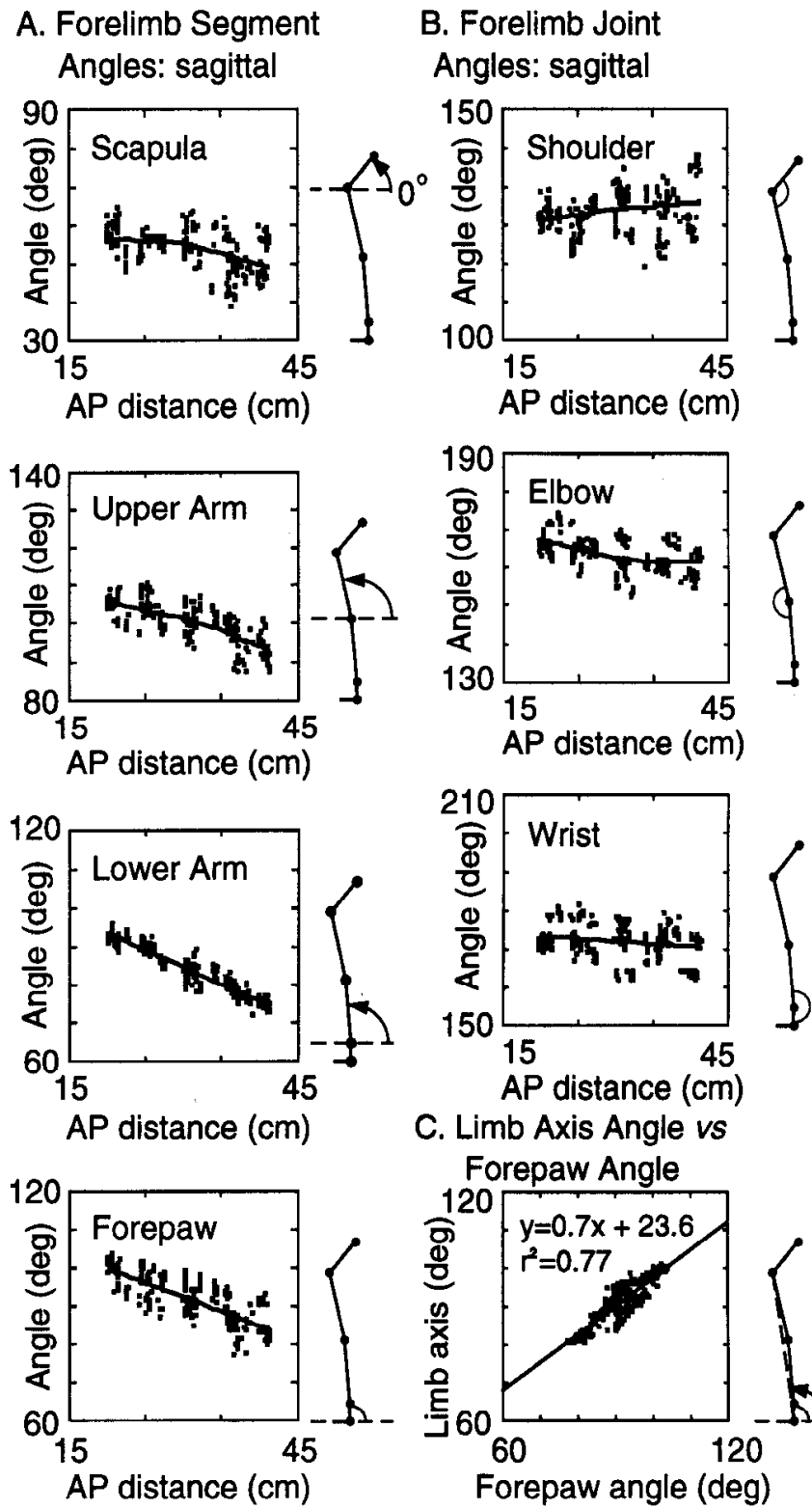

C. Limb Axis Angle vs

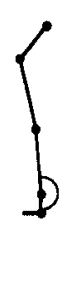

Forepaw Angle

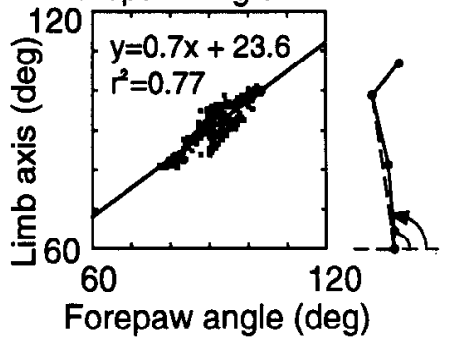

Figure 7. Stance kinematics of the forelimb. A, Sagittal segment angles plotted against AP distance, with superimposed LOWESS lines indicating linear relations. $B$, Sagittal joint angles plotted against AP distance with superimposed LOWESS lines. $C$, Direction of forelimb axis plotted against forepaw angle. Conventions are as in Figure 6.

lines through the scatter plots. Interestingly, the hip, knee, and ankle joint angles encompassed between the segments remained fairly constant with AP distance changes (Fig. 6B). Thus, limb configuration was generally maintained while the whole limb was levered forward or backward at the phalanges and the girdles (see also Figs. $2 A, 3 A$ ). In fact, the hindpaw segment angle could account for most of the variance in hindlimb axis orientation due to AP distance changes (Fig. $6 C$ ), with $r^{2}$ ranging from 0.81 to 0.93 in all four cats.

The forelimb also displayed linear changes in segment angles (Fig. 7A) and little change in joint angles (Fig. 7B), with AP distance changes. However, the range of segment angle changes was smaller than that observed in the hindlimb, primarily because the forelimb did not pivot as much as the hindlimb (see also Figs. 2A, 3A). The association between forelimb axis ori- 
A. Hindlimb Joint Torque: sagittal plane
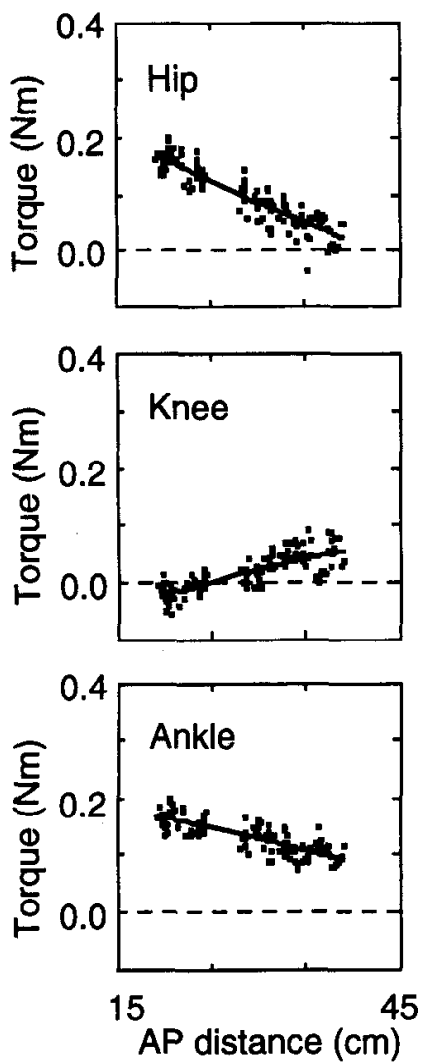

B. Forelimb Joint Torque: sagittal plane
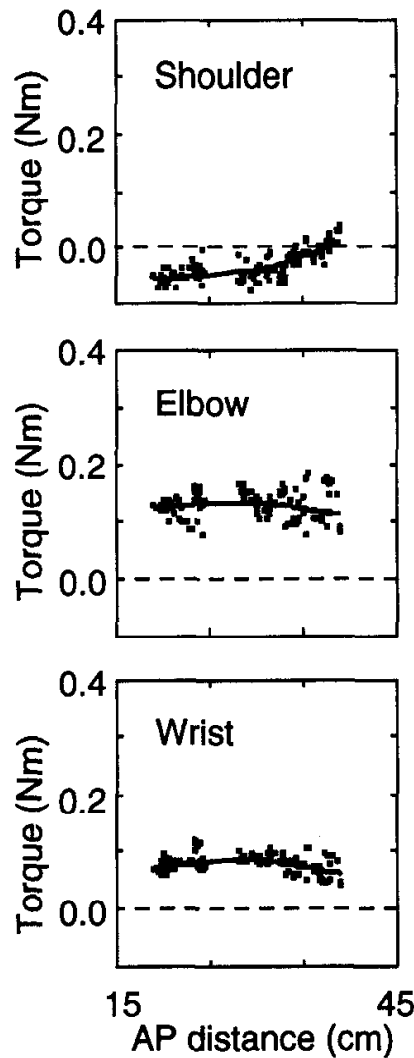

Figure 8. Sagittal plane joint torques of the hindlimb $(A)$ and forelimb $(B)$ from cat PR, plotted against AP distance. Positive values indicate extensor torque. LOWESS lines show linear trends in hindlimb joints and constant relations in forelimb joints with changes in AP distance.

entation and forepaw segment angle (Fig. $7 \mathrm{C}$ ) was also weaker, with $r^{2}$ ranging from 0.51 to 0.87 in all cats.

Joint torque. Figure 8 shows the sagittal plane joint torques of cat PR in the hindlimb and forelimb. At the shortest stance distance, there was a substantial amount of hip and ankle extensor torque and almost no knee torque (Fig. 8A). As stance distance increased, extensor torque decreased markedly in the hip and less so in the ankle, but increased at the knee. In the forelimb (Fig. 8B), there was a small amount of shoulder protractor torque, together with a moderate degree of elbow and wrist extensor torque, at the shortest stance distance. These were held fairly constant throughout distance changes except for a slight decrease of torque at each joint at the longest distance. Frontal plane (abduction) and horizontal plane (external rotation) joint torques increased slightly in the hindlimb as AP distance lengthened, while remaining fairly constant in the forelimb.

A cost function was computed by summing the squared 3-D torque from the hip, knee, ankle, and MTP joints, resulting in a curvilinear relation with AP distance changes. This function is related to the "effort" required in the maintenance of upright posture (Nubar and Contini, 1961). Figure 9A shows a U-shaped relation in this function in cats GR and MI, and an L-shaped relation in cats PR and LU. A one-way ANOVA was performed in each cat, using the cost function as the dependent variable and fore-hindpaw distance as the independent variablc with three levels (shortest, preferred, and longest). All four cats

A. Hindlimb
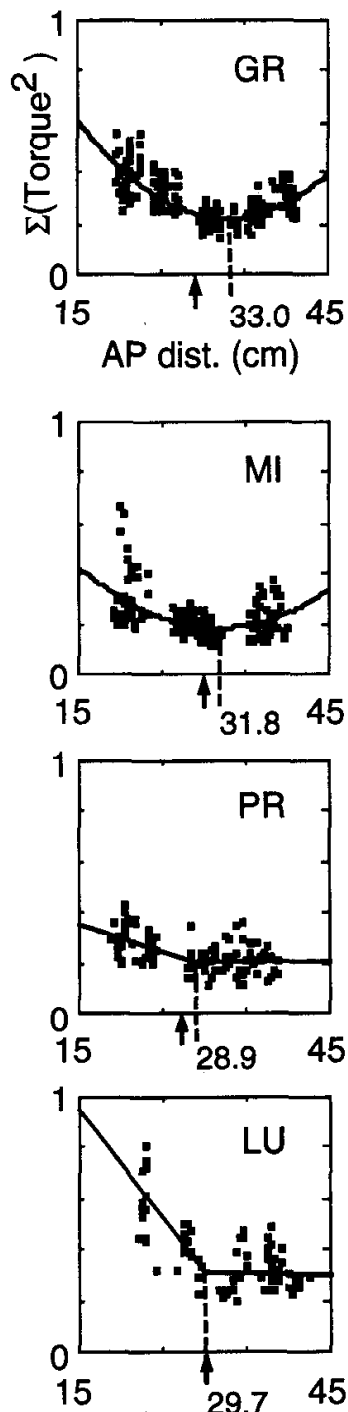

B. Forelimb
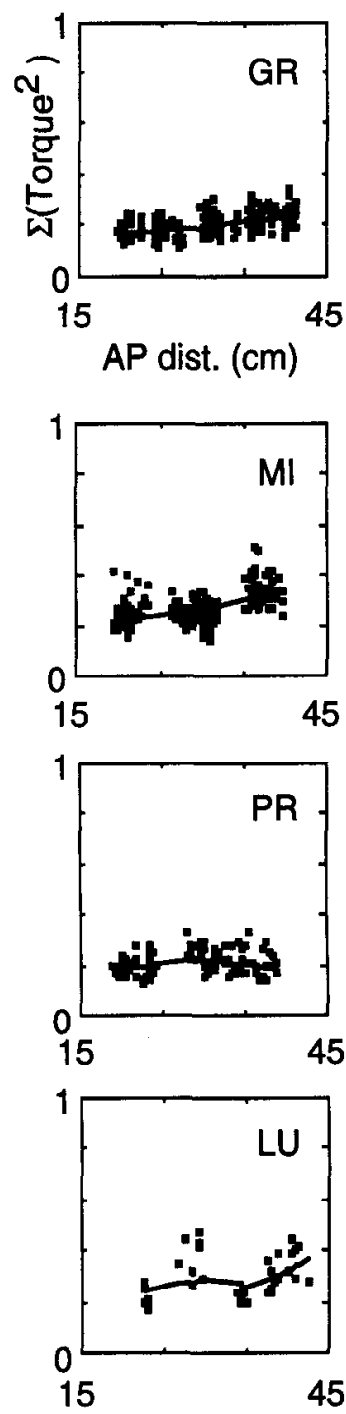

Figure 9. The cost function (sum of squared 3-D joint torques) for the hindlimb $(A)$ and forelimb $(B)$ versus AP distance in all four cats. Curve fitting was performed for the hindlimb cost function $(A)$ to find the distance corresponding to the minimal effort (indicated as dotted lines). Arrows indicate the preferred stance distance $(29,30,27$, and 30 $\mathrm{cm}$ for the four cats, respectively) observed during floor behavior. LOWESS lines in the fore $(B)$ indicate a general trend of conservation of effort.

showed a significant main effect due to fore-hind distance changes. Post hoc Tukey test of pairwise comparisons showed that, in cats GR and MI, the mean cost function at the preferred distance was significantly lower than that at the shortest or the longest stance distance. In cats PR and LU, the mean cost functions at the preferred and the longest stance were both significantly lower than the mean at the shortest distance, but the two were not significantly different from each other. Thus, a parabola curve was fitted to the data of GR and MI, while two linear regression lines were fitted to the data of $P R$ and $L U$. The fitting was done using a nonlinear model of parameter estimation, with the resulting parabola vertex or the turning point of two slopes indicating the fore-hind distance at which the cost function was minimal (see dotted lines in Fig. 9A). This distance was re- 
A. Average tonic EMG activity

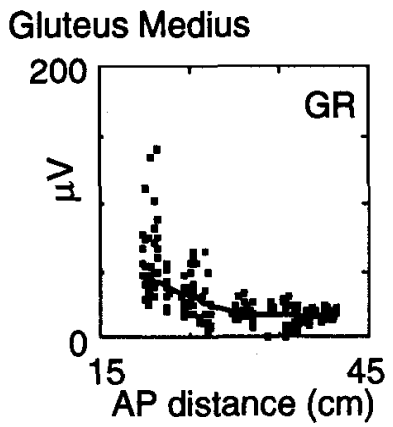

Vastus Lateralis

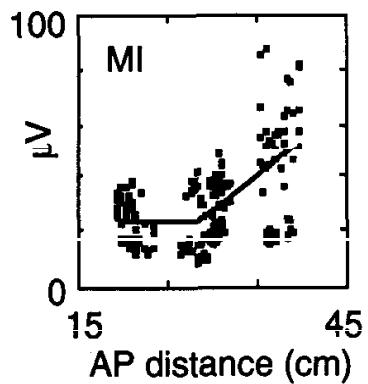

Soleus

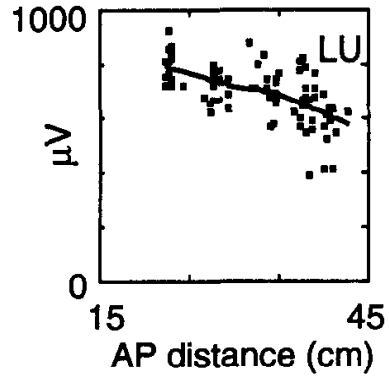

Iliocostalis

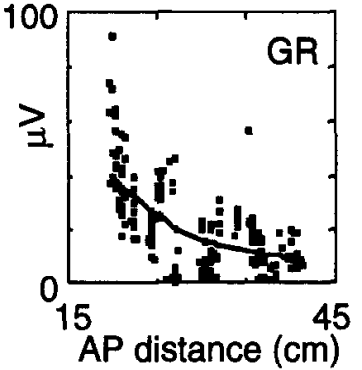

Longissimus Dorsi

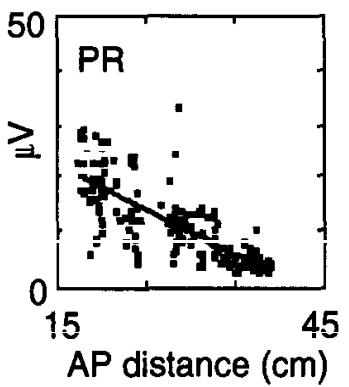

Lat. Gastrocnemius

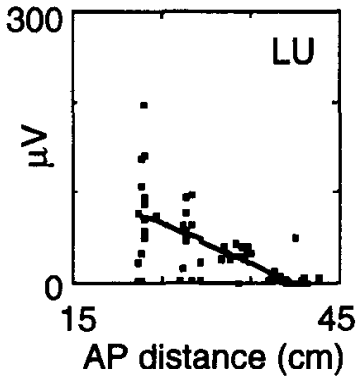

B. Joint Torque vs EMG
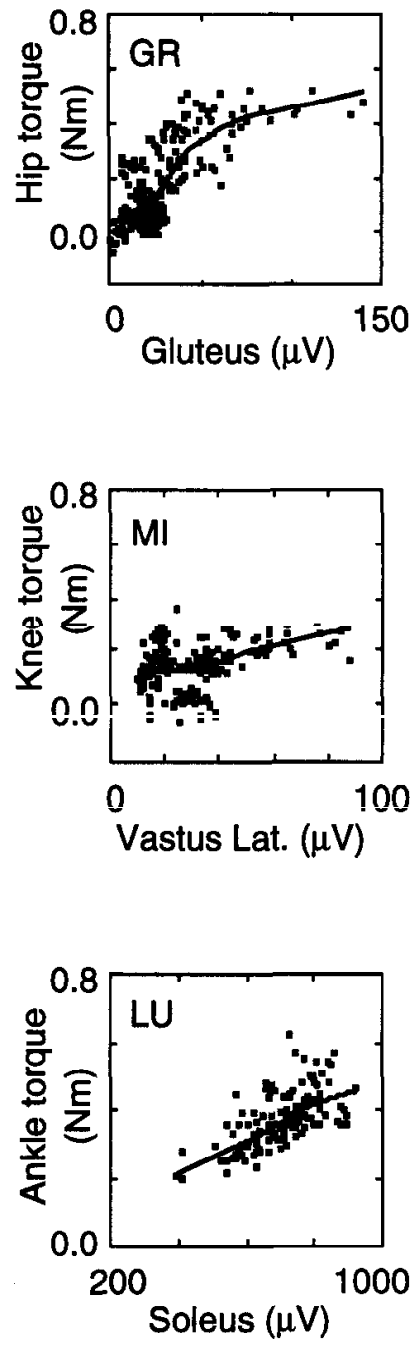

Figure 10. A, Tonic EMG activity during stance from selected trunk and hindlimb extensors plotted against AP distance. $B$, Joint torque plotted against extensor EMG activity. markably similar to the "preferred" stance distance (see arrows in Fig. 9A) for each cat, and corresponded to the length of the trunk axis. The horizontal GRF for the hindlimb at this distance was diagonally directed (ranging from $32.6^{\circ}$ to $49.5^{\circ}$ ), giving rise to a compressive force along the long axis of the trunk.

In the forelimb, the cost function remained relatively constant with AP distance changes. Results from the ANOVAs and post hoc comparisons showed no significant differences, except at the longest distance in cats $\mathrm{MI}$ and LU where the cost function was significantly larger than that at the preferred or the shortest stance distance.

Tonic background EMG activity. Among all the muscles implanted, tonic activity was consistently present only in soleus, other hindlimb and back extensors being active only at certain AP distances, while the flexors were invariably silent. Figure $10 \mathrm{~A}$ shows examples of the average background EMG activity from all the quiet stance trials plotted against AP distance. High gluteus activity was present at the shortest distance, and decreased as AP distance increased. Soleus and lateral gastrocnemius showed linear trends of decrease as AP distance increased, with soleus showing substantial activity even at the longest stance distance. In contrast, vastus lateralis showed an opposite trend of no activity at shortest stance and increasing activity as AP distance increased. The lumbar extensors, iliocostalis and longissimus dorsi, showed a trend of decreased activation with increased AP distance.

Although there was much variability in extensor muscles, they could generally account for some of the joint torque changes observed as stance distance changed (Fig. 10B). In the hip, extension torque was related to gluteus activity, with a linear trend of increase, saturating at higher levels of EMG activity. In the knee, there was little correlation of joint torque at lower levels of vastus activity, but the extension torque increased linearly with increased level of vastus activity. In the ankle, a linear trend of increased extension torque with increased soleus activity was present.

In summary, quiet stance at short fore-hind distances required increased muscular effort from the lower trunk and pelvis as well as the hip and ankle, whereas increased knee extensor activity was needed to maintain stance at longer fore-hind distances. For a cat to maintain posture on a horizontal surface, however, there were no circumstances in which stance could be achieved without muscular effort. 
A. Limb Axis Orientation: frontal plane
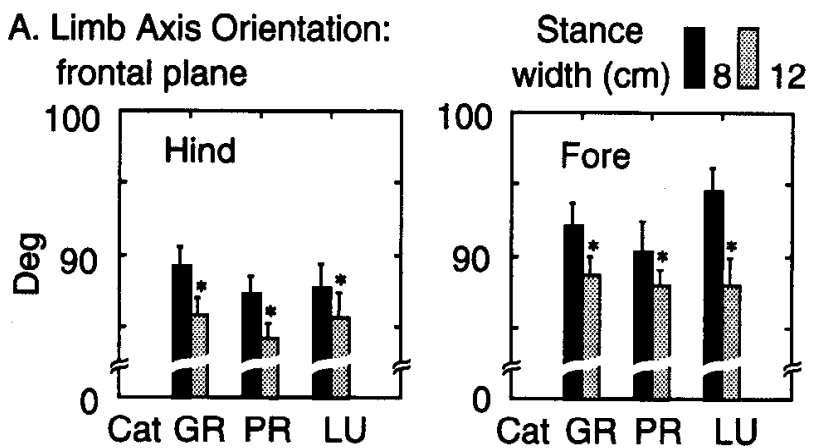

B. Force Vector Direction: frontal plane
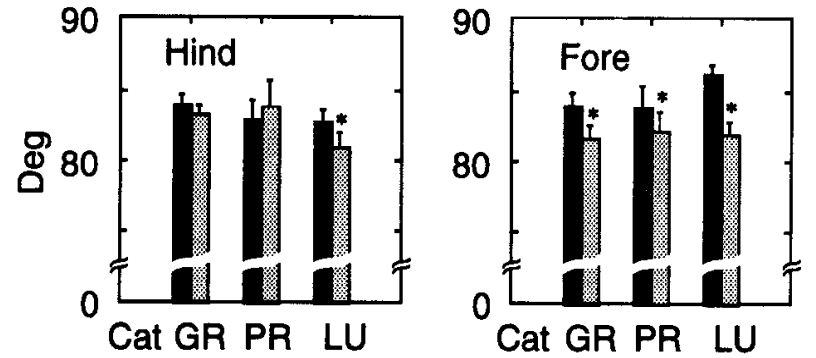

C. Force Direction vs Limb Axis Orientation
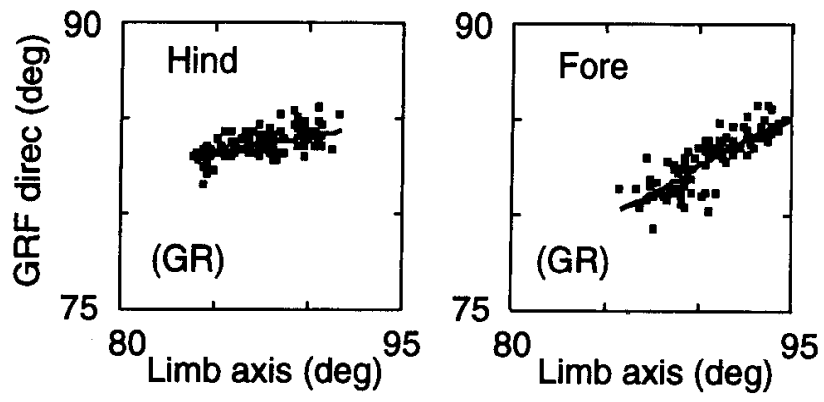

Figure 11. Effect of stance width on frontal plane limb axes and ground reaction forces. $A$, Average orientation of the frontal plane hindlimb and forelimb axes from the three cats standing at two widths, 8 $\mathrm{cm}$ (solid bars) and $12 \mathrm{~cm}$ (shuded bars). Error bars denole 1 SD. Significant differences $(p<0.01)$ are indicated by asterisks. $B$, Average orientation of the frontal plane force vectors. $C$, Frontal plane force vector direction plotted against limb axis orientation.

\section{Effects due to changes in stance width}

Due to the limitation of platform dimensions, only a small increment in width was tested. Thus, the observed changes in kinematics and forces due to increase in stance width (Fig. 11) were much smaller than those due to increase in fore hind distance. As width increased from 8 to $12 \mathrm{~cm}$, there was a significant decrease in limb axis orientation in the frontal plane for all cats in both the hindlimb and forelimb (Fig. 11A). Thus, the limbs were planted more outward as stance width increased, but the change was maximally $7^{\circ}$ and generally less than $4^{\circ}$. The GRF vector in the frontal plane (Fig. $11 B$ ) showed no change in the hind except for a small decrease in orientation in cat LU, whereas a small but significant decrease in orientation was observed in the forelimb in all cats. The decrease in GRF was even smaller than that observed in the limb axis. Figure $11 \mathrm{C}$ shows the relation of GRF direction to limb axis orientation. No linear relation existed in the hindlimb, with $r^{2}$ ranging from 0.01 in
PR to 0.37 in GR, whereas a moderate to strong linear relation was present in the fore, with $r^{2}$ ranging from 0.73 in PR to 0.93 in LU. Other than those illustrated, no significant changes in the magnitude of forces or joint torque were observed.

\section{Discussion}

In order to accommodate changes in interpaw distance, all cats adopted a strategy of using the limbs as levers with adjustable inclination while conserving trunk orientation and intralimb geometry. By inclining the ground reaction forces along with the limb axes, changes in joint torques werc minimized. A cost function estimating effort, based on the sum of squared joint torques, was optimized in the hindlimb at a stance distance similar to that preferred by each cat on the floor. These results were robust in that they were repeatable within the same cat as well as between cats. It is concluded that stabilization of the trunk in space is a major postural goal during stance.

The minimization of the cost function at or near the preferred stance distance suggests that the selection of a preferred distance may be primarily related to energetics. The actual distance is likely a function of the length of the trunk axis. According to the "principle of minimum effort" proposed by Nubar and Contini (1961), the most probable motion or posture adopted by the individual is one in which the joint torques minimize the cost function, E, while simultaneously satisfying some imposed constraints which may take the form of anatomical or structural limitations. In order to minimize joint torque in the standing cat, the force vector at the ground must remain closely aligned with the limb axis as stance distance changes, otherwise the muscle activation level and net torque will increase in one or more joints due to the change in moment arm length. Excessive loading could result, leading to the inability to maintain such a posture for long periods of time due to fatigue. The preferred distance for quadrupedal stance may have a similar connotation to the preferred speed adopted by animals during different gaits of locomotion. Alexander (1989) has shown that optimal gaits are adopted by different species of animals to minimize the energy expenditure for the range of speeds required. Hoyt and Taylor (1981) demonstrated that ponies, trained to move at a wide range of treadmill speeds, chose only narrow ranges of speed for walk, trot, or gallop when they were allowed to move freely. Their preferred speed within each gait coincided with the minimal volume of oxygen consumed to move a given distance.

Within the limitations of the present study, we propose that not only is there an optimal stance distance for the cat, but there is also an optimal postural orientation or body geometry, the configuration of which is related to energetics and morphometrics such as the length of the torso and the limb segments. By averaging the stance configurations and ground reaction forces from the four cats at their minimal hindlimb cost functions, an optimal body posture can be defined (Fig. 12), which consists of (1) a constant orientation of the trunk aligned parallel to the support surface; (2) vertical forelimb axis and inclined hindlimb axis with invariant intralimb joint angles (excluding those of the phalanges and girdles); and (3) inclined ground reaction forces resulting in a compressive force along the long axis of the trunk that may be functional for stabilizing the spinal column.

The strategy of maintaining a constant trunk and changing limb orientation to accommodate changes in stance distance is not obligatory. Cats are certainly capable of changing both the orientation of the limbs and the trunk simultaneously, as shown during galloping (Goslow et al., 1973). The choice of strategy 


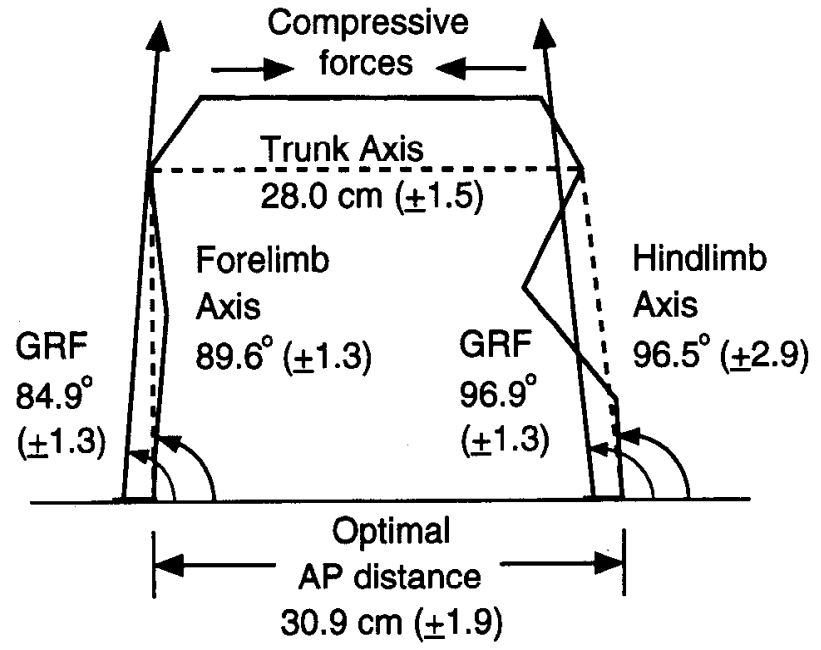

Figure 12. Optimal body geometry in the sagittal plane obtained by averaging from the four cats the angles of the limb axes and ground reaction forces at the AP distance which yiclded a minimal cost function in the hindlimb. The mean orientations of the limb axes and GRFs as well as the length of the trunk axis and optimal AP distance are shown with the SD in parenthesis. The optimal stance configuration consists of (1) horizontal alignment of the trunk, (2) vertical forelimb axis and slightly inclined hindlimb axis, and (3) inclined GRFs resulting in compressive forces along the longitudinal axis of the trunk.

is task dependent and also related to energetics. Although the trunk did not change its configuration as stance distance shortened, the epaxial extensors of the back showed an increased level of tonic activity. This may reflect a reduction in compression forces along the long axis of the vertebral column due to the change in inclination of the GRFs (Gray, 1944). The alternative strategy of arching the back could require an even greater activation of many back muscles and hence fatigue.

It is interesting that the hindlimb configuration and GRF alignment observed during quiet stance at the shortest AP distance is reminiscent of the posture seen during late stance in backward walking (Perell et al., 1993), where the fore-hindpaw distance is relatively short, as compared to forward walking. There is a marked change in knee torque from that of extensor to flexor during the stance phase of backward walking primarily due to activation of the medial gastrocnemius, as confirmed by tendon force output measurements. In the present study, the short stance distance was characterized by a decrease in vastus lateralis activity, as well as an increase in lateral gastrocnemius activity, at least during some trials, resulting in a minimal knee torque.

The smaller range of directional changes observed in the forelimb axis, coupled with its close relation with the GRF and the straighter alignment of the limb segments, may be indicative of a different functional role in the fore as compared to the hindlimb. The forelimbs may be used more as mechanical struts while the hindlimbs function as levers to accommodate the change in stance distance. This has also been suggested by an earlier study based on the active forces observed in response to horizontal translation of the support surface (Macpherson, 1988). There may exist a limit in the degree of limb angling that can bc tolcratcd, related in part to the surface coefficient of friction. Beyond that limit, the animals may have to change strategies in order to maintain stability. This could not be investigated due to the limited size of the platform.
Previous studies of cat posture (Lacquaniti et al., 1984, 1990) showed that cats accommodated changes in the inclination of the support surface primarily by rotating at the proximal and distal limb joints, similar to our findings for stance distance changes on a horizontal surface. As the support tilt changed, the trunk remained parallel to the support surface, whereas the limb axes remained oriented vertically. The authors concluded that the main controlled variable in cat postural orientation was the limb axis and, furthermore, that this axis was oriented to the external environment (namely, earth's vertical). In light of the results from both studies, we conclude instead that trunk alignment, rather than limb axis orientation, is the main controlled variable for stance in cats. That is, that trunk length is constrained and trunk axis is oriented to the external variable of surface inclination. Our study and Lacquaniti's (Lacquaniti et al., 1984, 1990) together show that trunk axis and intralimb geometry are postural constraints of quadrupedal stance, and support the view that controlling the orientation of the trunk in space is a primary goal of the postural control system.

The idea of a preferred postural orientation is intriguing because it truly involves multidimensional sensorimotor integration by the central nervous system. In order to stabilize oneself in space, certain aspects of body geometry must be controlled or constrained. This involves orientation with reference to visual, vestibular, proprioceptive, and tactile information, as well as synthesis of all the available information to make adjustments in the multisegmented musculoskeletal systerm. Thus, there exists a constant interaction between the body axes and some external reference frame. Gurfinkel et al. (1981) have shown that, in humans, stabilization of the trunk with respect to earth's vertical is critical in the maintenance of upright stance.

How is trunk orientation detected for control? Proprioceptors in the trunk, particularly in the muscles of the vertebral column, as well as joint receptors may all contribute to the detection of the forces acting on the trunk, including both compressive and gravitational forces. These signals could contribute to the spatial perception of trunk orientation. The strategy of maintaining a constant intralimb geometry of the four limbs is sufficient to maintain the trunk height and orientation to the support surface. Length and force changes in the limb muscles are detected by Ia and $\mathrm{Ib}$ afferents, respectively, while cutaneous and deep mechanoreceptors in the paw pads may also be important in conveying information regarding the nature of the support surface.

By expanding on Bernstein's hierarchical principle of motor control (Bernstein, 1967), Gurfinkel and Levick (1991) have advanced the notion that there is an internal representation of a general body scheme, or postural orientation. They hypothesized that postural control consisted of a lower level of automatic responses operating with only partial information available, and a higher level of CNS control operating on global information to form an internal body representation in space. This hypothetical body scheme forms the basis of conscious perception of body configuration, as well as orientation with respect to extrapersonal space and the formation of a task-dependent reference system. The reason for orienting the trunk instead of other body parts in stance may be due to the fact that the trunk contains the greatest mass. Based on psychophysical reports of the estimated vertical in humans under varying surrounding conditions, it has been suggested that the CNS uses a weighted combination of labyrinthine gravitoinertial signals, as well as visual and somatosensory cues, to stabilize the trunk in space (Mittelstaedt, 1983; Young 
et al., 1984). It was further hypothesized that a state vector would be computed in the spatial orientation process, based on the estimated position of the trunk from the vertical, to use the trunk as an egocentric reference frame in determining body positions with respect to external objects. This is corroborated by Mouchnino et al. (1993), who showed that the trunk was used as a reference frame for the performance and internal calibration of leg movements in both trained and untrained dancers. The only difference was that trained dancers oriented their trunks to vertical while untrained dancers oriented only their heads and inclined their trunks, but the actual hip abduction angles relative to the trunk remained the same.

\section{References}

Alexander RMcN (1989) Optimization and gaits in the locomotion of vertebrates. Physiol Rev 69:1199-1227.

Bernstein N (1967) The coordination and regulation of movements. Oxford: Pergamon.

Goslow GE, Reinking RM, Stuart DG (1973) The cat step cycle: hindlimb joint angles and muscle lengths during unrestrained locomotion. J Morphol 141:1-42.

Gray J (1944) Studies in the mechanics of the tetrapod skeleton. J Exp Biol 20:88-116.

Gurfinkel VS, Levick YS (1991) Perceptual and automatic aspects of the postural body scheme. In: Brain and space (Paillard J, ed), pp 147-162. London: Oxford UP.

Gurfinkel VS, Lipshits MI, Mori S, Popov KE (1981) Stabilization of body position as the main task of postural regulation. Fiziol Chelov $7: 400-410$.

Horak FB, Nashner LM (1986) Central programming of postural movements: adaptation to altered support-surface configurations. J Neurophysiol 55:1369-1381.

Hoy MG, Zernicke RF (1985) Modulation of limb dynamics in the swing phase of locomotion. J Biomech 18:49-60.

Hoyt DF, Taylor CR (1981) Gait and the energetics of locomotion in horses. Nature 292:239-240.

Lacquaniti F, Maioli C, Fava E (1984) Cat posture on a tilted platform. Exp Brain Res 57:82-88.

Lacquaniti F, le Taillanter M, Lopiano L, Maioli C (1990) The control of limb geometry in cat posture. J Physiol (Lond) 426:177-192.

Macpherson JM (1988) Strategies that simplify the control of quadrupedal stance. 1. Forces at the ground. J Neurophysiol 60:204-217.

Macpherson JM (1994) Changes in a postural strategy with inter-paw distance. J Neurophysiol 71:931-940.

Macpherson JM, Lywood DW, van Eyken A (1987) A system for the analysis of posture and stance in quadrupeds. J Neurosci Methods 20:73-82.

Mittelstaedt $H$ (1983) A new solution to the problem of the subjective vertical. Naturwissenschaften 70:272-281.

Mouchnino L, Aurenty R, Massion J, Pedotti A (1993) Is the trunk a reference frame for calculating leg position? Neuroreport 4:125-127.

Nubar Y, Contini R (1961) A minimal principle in biomechanics. Math Biophysiol 23:377-391.

Perell KL, Gregor RJ, Buford JA, Smith JL (1993) Adaptive control for backward quadrupedal walking. IV. Hindlimb kinetics during stance and swing. J Neurophysiol 70:2226-2240.

Roberts TDM (1973) Reflex balance. Nature 244:156-158.

Young LR, Oman CM, Watt DG, Money KE, Lichtenberg BK (1984) Spatial orientation in weightlessness and readaptation to earth's gravity. Science 225:205-208. 